\title{
Working the night shift: a necessary time for training or a risk to health and safety?
}

\author{
${ }^{1}$ I Morrison, ${ }^{2} \mathrm{D}$ Flower, ${ }^{3} \mathrm{~J}$ Hurley, ${ }^{4} \mathrm{RJ}$ MacFadyen \\ ${ }^{1}$ Consultant Neurologist, Department of Neurology, Ninewells Hospital, Dundee, and Consultant Sleep Medicine, Department of Sleep \\ Medicine, Royal Infirmary of Edinburgh, Edinburgh, Scotland; ${ }^{2}$ Senior Health Director, Safety and Operational Risk, BP International Ltd, \\ Middlesex, England; ${ }^{3}$ Director of Physician Training and Consultant Physician, Division of Internal Medicine, Ballarat Base Hospital and \\ University of Melbourne, Rural Clinical School, Victoria, Australia; ${ }^{4}$ Director of Internal Medicine and Consultant Cardiologist, Division of \\ Internal Medicine, Ballarat Base Hospital and University of Melbourne, Rural Clinical School, Victoria, Australia
}

\begin{abstract}
The European Working Time Directive (EWTD) limits excessive night shifts and restricts the working week to no more than 48 hours. The underlying rationale is to minimise the health risks to all workers. Here we debate the impact of night rotas for doctors-in-training on patient safety and medical education; when the EWTD was agreed these topics may not have been considered, either systematically or objectively. The impacts of diurnal rhythms on human functions affect all night workers, but the nature of rostered medical and surgical work has little precedent in other industries or even in the contracts of other healthcare staff. For example, rostered night duties need to be distinguished from permanent night shift work. On-call medical night work from training doctors is generally required for short periods and usually involves fewer patients. It is an important time in training, where clinical responsibility and decision-making can be matured in a supervised setting. To comply with the EWTD most hospitals have adopted rota patterns that aim to cover the clinical needs, while ensuring no doctor works for more than 48 hours in an average working week. To monitor this process longterm studies are necessary to evaluate effects on a doctor's health and on patient care generally. The EWTD has also led to a loss of continuity of patient care; does this really matter?
\end{abstract}

KEYWORDS Circadian rhythms, hospital night shift rotas, light-dark cycles, medical decision-making, sleep deprivation, sleep-wake biology

DECLARATION OF INTERESTS Robert McFadyen is the Clinical Editor of The Journal of the Royal College of Physicians of Edinburgh.

\author{
Correspondence to I Morrison \\ Department of Neurology \\ Ninewells Hospital \\ Dundee DDI 9SY \\ tel +44(0) I 382740034 \\ e-mail ian_m@doctors.org.uk \\ Correspondence to RJ MacFadyen, \\ Ballarat Base Hospital \\ PO Box 577, Ballarat 3353 \\ Victoria, Australia
}

tel +6I 53206974

e-mail Robertmac@bhs.org.au

\section{Human physiology should define hospital rotas, not training requirements}

I Morrison, D Flower

The European Working Time Directive (EWTD) was introduced in 1996 to limit the working week to no more than 48 hours, in addition to guaranteeing a minimum number of holidays, rest breaks and limiting excessive night shifts. Junior doctors in training were initially exempt from the EWTD, but a phased introduction, known as the 'New Deal', was negotiated by the British Medical Association to limit the impact on hospital services and training. This process was finally completed in 2009.,2 In order to meet the demands of these restrictions, hospital rotas inevitably moved towards a shift-working pattern for most junior medical staff. Overnight shifts can typically last 12 hours and while breaks are possible if the shift exceeds six hours, the minimum requirement for breaks is only 20 minutes. Junior staff are also expected to remain awake during their night shift, with on-call bedrooms being removed by many hospitals, a move opposed by the Academy of Royal Colleges and the British Medical Association. ${ }^{3}$

\section{THE IMPACT OF WORKING NIGHT SHIFTS}

\section{Medical errors and performance}

Medical trainees, however, have been shown to ignore these regulations and at times do not have sufficient recovery sleep from night shift. ${ }^{4}$ It has been shown that sleepy doctors are more likely to make medical 
errors. ${ }^{5-8}$ Chronic sleep deprivation, as occurs with shift working patterns, has also been shown to cause impairment of neurobehavioural performance in physicians.' Worryingly, many workers may be significantly impaired by sleep deprivation or fatigue, but have no awareness of their limitations. ${ }^{10}$

\section{Health}

Beyond the issues of patient safety, shift working has been shown to have an impact on the health of workers. Although there are virtually no studies from medical settings, data from other industries suggest that chronic circadian misalignment may be associated with a number of co-morbidities including cardiovascular disease, metabolic disturbances, gastrointestinal problems, fertility issues, mood disorder and some types of cancer." Mortality is also higher in those with chronic sleep restriction ${ }^{12}$ and driving while sleepy, particularly among junior doctors who often have lengthy commutes during their specialty training, is particularly dangerous. ${ }^{13}$

\section{SLEEP DEBT}

It is inevitable that working night shifts will lead to fatigue and sleepiness. Sleeping during the day is less efficient and restorative than sleeping overnight, and it has been shown that workers on night shift have significantly less sleep, with increasing 'sleep debt' with successive night shifts. ${ }^{14,15}$ This sleep debt that can be as much as two hours per day. Chronic sleep loss of this type can lead to significant performance impairment, ${ }^{16}$ with each hour of sleep lost being 2.7 times more potent than a gram per kilogram of alcohol ingested during studies. ${ }^{17}$ Over the course of a 'four-night' shift pattern, this is the equivalent to a whole night's sleep loss which, acutely, is comparable to a blood alcohol concentration of $100 \mathrm{mg} / 100 \mathrm{ml}$, well over the UK driving limit. ${ }^{18}$ The extent of this sleep deprivation is greater when trainees are required to work for seven night shifts in a row.

Physiological mechanisms also make it very challenging to work overnight. Daily variations in levels of wakefulness and sleepiness in the normal population are the result of two neurobiological mechanisms - the homeostatic mechanism where the pressure for sleep builds through the day and is dissipated by sleep and the circadian rhythm, which acts as an internal 'body clock' that promotes wakefulness during the day and sleepiness at night. ${ }^{19}$ These mechanisms are synchronised under normal, daylight working patterns. However, shift working can alter the usual pattern of sleep onset or wakening, desynchronize these two mechanisms, and cause patterns of chronic sleep loss resulting in continuous, partial sleep deprivation and increased sleepiness while awake, even if the worker has sufficient opportunity for sleep. ${ }^{20}$ Additionally, the circadian drive towards wakefulness is at its maximum during the day, and its lowest overnight. Working overnight therefore takes place when the drive for wakefulness is at its lowest, and this can contribute significantly to symptoms of fatigue and sleepiness when working night shift, with corresponding functional impairment. ${ }^{2 !}$

\section{ROTA PATTERNS}

Rota patterns are also a factor that increases fatigue. Despite this, there have been few studies looking at the optimum shift design that limits fatigue, reduces handover frequency and is also compliant with working time limitations. ${ }^{22}$

Many rotas will move staff from day shift immediately to a night shift. Many also do not allow for sufficient recovery periods from night shifts. It has been shown that performance remains impaired, even after three nights of recovery sleep. Rotas may allow their workers only two nights of recovery sleep before further nights shifts, or moving back to day shift, and this is clearly insufficient. ${ }^{16}$

Sudden changes to rota patterns are not advised as it impairs the worker's ability to adapt to night shift patterns. In industry, most transitions provide at least a 24-hour break between completing the day shift, and starting the night shift. This allows for a full night time sleep and afternoon nap prior to the nightshift and thus reduces the period of continuous wakefulness. Changing shift patterns in a 'clockwise' pattern are also thought to be beneficial in improving adaptation to working nights. ${ }^{11,14}$

\section{ADAPTATIONS}

Many hospitals have rotas where staff will work seven 12-hour shifts in a row. These patterns are often used to allow workers to adapt during their block of nights. Although adaptation to night shifts is, in theory, possible with time, it is very challenging and involves controlled use of light and behavioural methods. These techniques are demanding and, with the exception of small groups of workers in the oil industry who work off-shore in controlled conditions, prolonged blocks of nights is generally avoided in industry as a result.

Regardless, junior medical staff usually work relatively short blocks in an attempt to maximise training opportunities during the day and these are often too short for adequate adaptation, even with judicious use of light and other counter measures. ${ }^{13,23}$

Even with sufficient adaptations in the workplace, evidence suggests that certain individuals are incapable of adapting to working night shifts and may develop chronic sleepiness and/or insomnia as a result. This will be associated with increased functional impairment..$^{24,25}$ The International 
Classification of Sleep Disorders version 2 (ICSD-2) recognises this condition as shift work sleep disorder and estimates its prevalence as $\mathbf{2 - 5 \%}$ of the adult population, ${ }^{21}$ with other studies suggesting that $10 \%$ of night shift workers may experience this disorder. ${ }^{26}$

\section{CONCLUSION}

Night shifts are an inevitable consequence of the EWTD and come with inherent risk. They are clearly better than an on-call system where staff could work in excess of 120 hours per week and provide excellent opportunities for training. However, there is sufficient evidence, particularly from industry, to suggest that 'compressing' night shifts into blocks of four nights or greater, with shifts lasting 12 hours, does itself pose a risk.

\section{REFERENCES}

I British Medical Association. Implications for health and safety of junior doctors' working arrangements [Internet]. London: BMA; 2006 [cited 2013 July 3]. Available from: http://www.bma.org.uk/

2 Department of Health. European working time directive [Internet]. London: DOH; 2010 [cited 2013 July 3]. Available from: http:// www.nhsemployers.org/PlanningYourWorkforce/Medical Workforce/EWTD/Pages/EWTD.aspx

3 British Medical Association. Junior Doctors Committee and Academy of Medical Royal Colleges'Trainees' Committee. Joint position statement on on-call rooms [Internet]. London: BMA; 2006 [cited 2013 July 3]. Available from: http://www.asit.org/assets/documents/JDC_Ao MRC_oncallrooms.pdf

4 Fletcher KE, Underwood W 3rd, Davis SQ et al. Effects of work hour reduction on residents' lives: a systematic review. JAMA 2005; 294: I088-100. http://dx.doi.org/I0.100 I/jama.294.9.1088

5 Baldwin DC Jr, Daugherty SR, Tsai R et al. A national survey of residents' self-reported work hours: thinking beyond specialty. Acad Med 2003; 78:I I54-63. http://dx.doi.org/l0.1097/0000I8882003II000-00018

6 Landrigan CP, Rothschild JM, Cronin JW et al. Effect of reducing interns' work hours on serious medical errors in intensive care units. N Engl J Med 2004; 35I:I838-48. http://dx.doi.org/I0.1056/ NEJMoa04I406

7 Philibert I.Sleep loss and performance in residents and nonphysicians: a meta-analytic examination. Sleep 2005; 28:1392-402.

8 Ayas NT, Barger LK, Cade BE et al. Extended work duration and the risk of self-reported percutaneous injuries in interns. JAMA 2006; 296: 1055-62. http://dx.doi.org/I0.100I/jama.296.9.1055

9 Anderson C, Sullivan JP, Flynn-Evans EE et al. Deterioration of neurobehavioral performance in resident physicians during repeated exposure to extended duration work shifts. Sleep 2012; 35: I 137-46.

10 Van Dongen HP, Maislin G, Mullington JM et al.The cumulative cost of additional wakefulness: dose-response effects on neurobehavioral functions and sleep physiology from chronic sleep restriction and total sleep deprivation. Sleep 2003; 26: I I7-26.

II Shift work and sleep: optimizing health, safety, and performance. J Occup Environ Med 20I I; 53(5 Suppl):SI-10; quiz SI I-2.

12 Cappuccio FP, D'Elia L, Strazzullo $P$ et al. Sleep duration and allcause mortality: a systematic review and meta-analysis of prospective studies. Sleep 2010; 33:585-92.
Although this may be considered to be an inevitable consequence of shift working on training, we feel that patient and doctor safety should be the priority, and the effects on performance caused by night shift should be limited as far as possible. In particular, we call for a maximum of $2-3$ night shifts at any time, with a minimum of two nights recovery between these blocks.

We recognise that this will have an impact on training, but the evidence is clear - the experience from industry cannot be ignored and human physiology should be used as the guide to set rota patterns.

I3 Morrison I, Riha R, Douglas $\mathrm{N}$ et al. Dangers of driving after night shifts. BMJ Careers 2012.

14 Lerman SE, Eskin E, Flower DJ et al. Fatigue risk management in the workplace. J Occup Environ Med 20I2; 54:231-58. http://dx.doi. org/I0.1097/JOM.0b013e318247a3b0

I5 Folkard S, Lombardi S, Tucker PT. Shift work, safety, sleepiness and sleep. Ind Health 2005; 43:20-3. http://dx.doi.org/I0.2486/ indhealth.43.20

16 Borbely AA. A two process model of sleep regulation. Hum Neurobiol 1982; 1:195-204.

17 Van Dongen HP. Shift work and inter-individual differences in sleep and sleepiness. Chronobiol Int 2006; 23:1/39-47. http://dx.doi. org/l0.1080/07420520601 I0097I

I8 American Academy of Sleep Medicine. The international classification of sleep disorders. Diagnostic and coding manual. 2nd ed. Westchester, IL:American Academy of Sleep Medicine; $200 \mathrm{I}$.

19 Belenky G,Wesensten NJ,Thorne DR et al. Patterns of performance degradation and restoration during sleep restriction and subsequent recovery: a sleep dose-response study. J Sleep Res 2003; I 2: I-1 2.http://dx.doi.org/I0.1046/j. I 365-2869.2003.00337.x

20 Roehrs T, Burduvali E, Bonahoom A et al. Ethanol and sleep loss: a 'dose' comparison of impairing effects. Sleep 2003; 26:98I-5.

21 Dawson D, Reid K. Fatigue, alcohol and performance impairment. Nature 1997; 388:235. http://dx.doi.org/I0.1038/40775

22 Reed DA, Fletcher KE,Arora VM. Systematic review: association of shift length, protected sleep time, and night float with patient care, resident' health, and education. Ann Intern Med 2010; I53:829-42. http://dx.doi.org/I0.7326/0003-48I9-153-12-2010I2210-00010

23 RevellVL, Eastman $\mathrm{Cl}$. How to trick mother nature into letting you fly around or stay up all night.J Biol Rhythms 2005; 20:353-65. http:// dx.doi.org/ I0. I I77/0748730405277233

24 Van Dongen HP, Belenky G. Individual differences in vulnerability to sleep loss in the work environment. Ind Health 2009; 47:5 I8-26. http://dx.doi.org/I0.2486/indhealth.47.518

25 Shen J, Botly LC, Chung SA et al. Fatigue and shift work.J Sleep Res 2006; I5: I-5. http://dx.doi.org/ I0.I I I I/j. I365-2869.2006.00493.x

26 Drake CL, Roehrs T, Richardson G et al. Shift work sleep disorder: prevalence and consequences beyond that of symptomatic day workers. Sleep 2004; 27: 1453-62. 


\title{
The influence of sleep cycles on night medical work: understood in principle but of unclear significance
}

\author{
J Hurley, RJ MacFadyen
}

\section{CHRONOBIOLOGY AND TIME-SHIFTED WORK IS NOT NEW}

The circadian clock affects many aspects of human physiology and function and chronobiology has been recognised for nearly 100 years in a large body of scientific literature.' That night work efficiency is affected by this phenomenon in those who are not fully timeshifted to an alternate light-dark cycle is neither a recent finding nor contestable. ${ }^{2-4}$ That long periods of night working are associated with adverse health consequences for those that follow such work patterns long-term is also a well-established association. ${ }^{5}$ We agree that the extent to which this should impact on how medical services and junior doctor training are provided and how this should be structured and monitored is a valid matter for detailed consideration. However, we would suggest that out of context inferences are unhelpful.

\section{NIGHT MEDICAL DUTY: SWINGS AND ROUNDABOUTS}

Globally, healthcare requires periods of night medical duty; this is inevitable despite being unpopular, inconvenient and generally avoided by many. Medical night work tends to be rostered and generally shortterm. It is usually completed adequately and has a training benefit for junior doctors during a formative phase of their career. Permanent night shift is exceedingly rare in adult hospital medical practice in contrast to other contexts. With this in mind, it is worth considering this question from two aspects: work practices in the contemporary context and training imperatives.

The impact of brief naps on medical effectiveness is unclear, thus criticisms of expecting night working to be continuous (without sleep) in principle in staff who are not following an on-call pattern are sadly unsupported by data. The intensity of night medical work is linked with undeniable diurnal human activity. Fewer patients are seen at night, generally there is a clear-cut seasonal flow and, for many acute illnesses, a diurnal variation in disease onset is evident. The need for full capacity in the early hours is reduced accordingly. Actual demand or patient choice is rarely factored in. Finally and again without parallel in other industries, night working in medicine has been traditionally recognised as a time where medical staff in training can have some limited autonomy for a brief period and learn to manage patients in a less pressured environment and with support available on or off site. The training advantages of this should not be dismissed out of hand. There are some physician trainees who relish this prospect, some who find it difficult and a source of understandable anxiety and there are also yet others who discover their true capacity to make independent decisions only in this context. Avoiding decision-making and deferring responsibility is not a desirable learning algorithm.

\section{ROSTERED DUTY IN THE CONTEMPORARY CONTEXT}

Few people choose to work at night due to the inevitable social disruption this entails. Few will ever become fully time-shifted to a reversed sleep/wake cycle as routine night duty rosters tend to be short. A full reversal would take in excess of eight days of time, with gradual shifting. This is not standard practice and its value in terms of medical decision-making capacity is uncertain. However, it could easily be studied and compared with a control group without difficulty. Such studies would help to define any advantages either in medical work or overall wellbeing.

Much of the 'evidence' of the detrimental effects of medical night working comes from a former era of long continuous on-call periods, a time when 'resident' medical officer meant resident at the hospital, although not necessarily always busy. Most societies have long shifted their doctors away from such duty rosters and the residents quarters have been closed. Encouraging night staff to nap in such areas during continuous duty is an interesting concept to offset sleep disordered function. However, in a medical context this has no evidence base and is likely to be counterproductive in principle and practice. The impact of former continuous duty on medical intern staff, by forcing patterns of sleep deprivation or at best fractured sleep, have shown documented distortion of perception, temporal disorganisation and memory impairment and a broad range of mood changes linked to fatigue. ${ }^{6}$ The relevant questions in the contemporary context are the extent of impaired biology from poorly synchronised rostered night working and quantifying the impact of this, if any, on medical efficiency and effectiveness.

\section{DETRIMENTAL: BY WHAT MEASURE?}

Claiming that periods of rostered night working is harmful is an extrapolation of the undoubtedly evident physiological effects derived in different contexts. Actual data on how doctors work at night are lacking, although 
inferences from permanent night shift or other levels of work with no relation to medical decision-making are abundant. This is scientifically unsound. An important confounder in all of this is the uncertainties in work practice by doctors and possible manipulation of their own activity to fit with social agendas or personal commitments which result in exacerbation of sleep deprivation. This would be an entirely understandable behaviour, but it is difficult to factor in the overall rostering and employment of trainee doctors. It is not avoidable by changing the roster.

Medically qualified staff numbers have risen exponentially to cope with reducing on-call duty hours and replacing these with rostered duties and handover at considerable cost in every health economy. The impact of this shift in service provision on the quality and quantity of training time is unknown, but logically there would be a reduction in cumulative experience following directly from reduced training time and patient exposure. This is most easily quantified in surgical practice ${ }^{7}$ but is likely to be directly mirrored in medical training. Claiming the detrimental impact of curtailing night work is irrelevant is simply illogical.

Reduced training (by simply logging numbers of operative and procedural tasks done) is more readily defined for surgical training. However, this is harder to define in relation to the quality of medical decisionmaking for physician trainees. Anyone who has worked at night knows that there are unique challenges in this context. Would altering or avoiding such shifts result in better decision-making? This is unclear. The problem is that there is no real evidence of predicated outcome either way as the workload, nature and component parts of medical practice and decision-making have largely been left unstudied and unquantified. Suppositions about the impact are premature and are confounded within the wholescale adjustments of the undergraduate medical curriculum in the UK that have occurred in the past 30 years.

Medical working at night is about medical decisionmaking. Few appear to be able to recognise or define the importance of this activity, which is little researched in diurnal medicine, far less in night working. ${ }^{8}$ Presumptions of impending disaster linked to rostered night work are largely speculative. Comparison with other industries is problematic. For example, parallels to aviation are not simple. Pilot error can be minimised through repeated flight simulation during training prior to certification and automation of many tasks in flight. Simulation as a component of medical training is in its infancy and is devoted primarily to simple procedural tasks. The extent of cognitive and decision-making skills required by a physician is as numerous as there are patients who will present in the night shift.

\section{TIME FOR RESEARCH RATHER THAN COMMENT}

What value is there in further considering sleep-wake biology in medical rosters? Could service design be usefully altered? What then in principle is special about a medical shift worker (as most training doctors are).. We would suggest that the occult effects of their sleepwake cycle shift and neurobiology is to be balanced against acquiring diagnostic experience. Methods now exist for scientific study in this area. Studies that track activity and wakefulness could easily be done blinded by using anonymous GPS activity data from cell phones and accelerometers uploading continuously. Clearly, bias might be entered if the subject knew of their monitoring and, of course, phone movement is only a possible surrogate for wakefulness. Better technologies to monitor drowsiness during activity and work are available in many sophisticated automobiles,, 10 and individualised personal web browsers," collecting data on eye movement as well as facilitating web access. The use of such systems might better illustrate the impact of night working on awareness level.

Abrupt short-term on-call patterns are potentially detrimental if rostered to these and where diurnal patterns of activity are maintained. Long-term night work or on-call with sleep disturbance is now unusual. Individual manipulation of activity and attempts to covertly maintain a diurnal activity pattern would be commonly encountered. Clearly staff cannot be forced to time-shift and only encouraged to do so.

\section{CAN NIGHT DUTY BE IMPROVED?}

Manipulation of light-dark cycles has been adequately demonstrated in many neurophysiological studies for nearly 100 years. Clearly this can be achieved but at what cost? Is it worth the effort in a medical context? Weekly rotation of duties as is commonly employed in some institutions (for example, the rostering of other health workers such as hospital nursing staff) is undoubtedly problematic as phase shifting of activity and responsiveness will only occur by one or two hours a day, meaning that for most it is only by the end of the duty week that adjustment has occurred. At this point they are often placed back to day duty following a period of rest which is inadequate to restore them to a normal sleep-wake pattern.

\section{CONCLUSIONS}

Broader sleep-wake biology is undeniably relevant to rostered night work in medicine. The basis of this has been known for decades. Damaging on-call patterns with repetitive disturbed and prolonged periods on-call have been largely abandoned and reference to them is 
irrelevant to this issue. We have technologies to define and study medical work better in terms of wakefulness, impact on function and the relevance to decisionmaking. Moreover, the social pressures on training doctors need also to be recognised as factors in their overall work/life balance. We would suggest calling for information, understanding and relevant workplace observations using novel monitoring technologies rather than unscientific association and presumption.

\section{REFERENCES}

I Moore-Ede MC, Czeisler CA, Richardson GS. Circadian timekeeping in health and disease. Part I Basic properties of circadian pacemakers. N Engl J Med 1983; 309:469-76. http:// dx.doi.org/I0.1056/NEJMI 98308253090806

2 Mills JN, Minors DS, Waterhouse JM. The circadian rhythms of human subjects without timepieces or indication of the alteration of day and night. J Physiol 1974; 240:567-94.

3 Czeisler CA, Moore-Ede MC, Coleman RH. Rotating shift work schedules that disrupt sleep are improved by applying circadian principles. Science 1982; 217:460-3. http://dx.doi.org/I0.II26/ science.7089576

4 Taylor PJ, Pocock SJ. Mortality of shift and day workers 1956-1968. $\mathrm{Br} J$ Ind Med 1972; 29:201-8.

5 Freidman RC, Bigger JT, Kornfield DS. The intern and sleep loss. N Engl J Med 197I; 285:20I-3. http://dx.doi.org/10.1056/NEJM 197107222850405

6 Antiel RM, Reed DA, Van Arendonk KJ et al. Effects of duty hour restrictions on core competencies, education, quality of life, and burnout among general surgery interns. JAMA Surg 2013; I48:44855. http://dx.doi.org//0.1001/jamasurg.2013.1368
7 Sugden C, Housden CR, Aggarwal R et al. Effect of pharmacological enhancement on the cognitive and clinical psychomotor performance of sleep-deprived doctors: a randomized controlled trial. Ann Surg 2012; 255:222-7. http://dx.doi.org/10.1097/ SLA.0b013e3182306c99

8 Magosso E, Ursino M, Zaniboni A et al.Visual and computer-based detection of slow eye movements in overnight and 24-h EOG recordings. Clin Neurophysiol 2007; I I8:I |22-33. http://dx.doi. org/10.1016/j.clinph.2007.01.014

9 Daimler. Attention assist: drowsiness detection system warns drivers to prevent them falling asleep momentarily [Internet]. Germany: Daimler [cited 2013 July 24]. Available from: http://media.daimler. com/media/0-92 I-658892-I-I | 47698-I-0-0-I | 47922-0-I-I I 7020-0-I-0-0-0-0-0.html?TS=| 266506682902

10 Glass. Welcome to a world through glass [Internet]. Available from: http://www.google.com/glass/start/what-it-does/

\section{CONFERENCING AND EVENTS}

The Royal College of Physicians of Edinburgh has a unique blend of rooms providing the perfect location for your conference, meeting or event. The Victorian Great Hall, galleried New Library and the Georgian Cullen Suite are wonderful settings for dinners and receptions. The modern Conference Centre seats up to 300 people in raked seating and is complemented by breakout rooms seating from 10 to 150 people, a keypad voting system and video conferencing. The College provides a stunning setting for weddings and receptions and is licensed for both civil and religious ceremonies. Discounts are available for Fellows and Members, medical conferences and charities.

For more information or for a quotation, please contact the Events Team on +44 (0) I3। 225 7324; email events@rcpe.ac.uk or visit http://www.rcpe-venue.co.uk

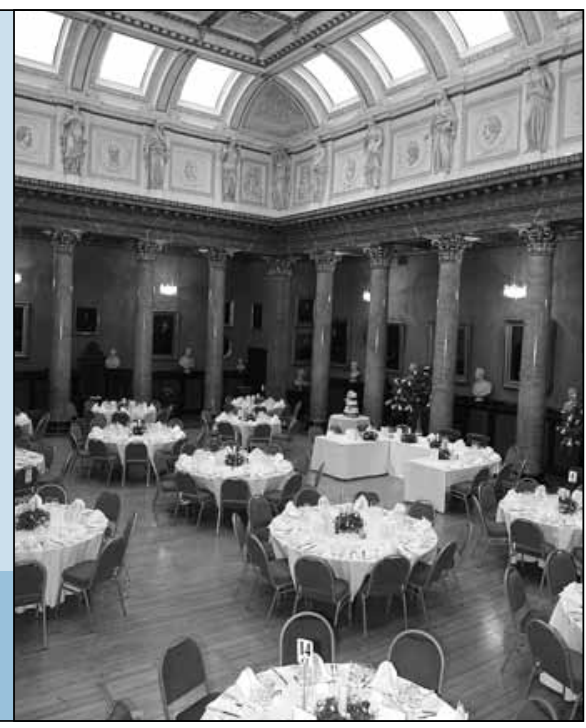

